\title{
Atom-Resolved Analysis of Birefringence of Nonlinear Optical Crystals by Bader Charge Integration
}

\author{
Chen-Sheng Lin, An-Yi Zhou, Wen-Dan Cheng, Ning Ye and Guo-Liang Chai* \\ State Key Laboratory of Structural Chemistry, Fujian Institute of Research on the Structure of Matter, Chinese \\ Academy of Sciences, Fuzhou 350002, P. R. China
}

Email: cslin@fjirsm.ac.cn

g.chai@fjirsm.ac.cn

We present an example of the calculation for $\mathrm{MgF}_{2}$ crystal using this atom-resolved analysis method. The experimental crystal structure (Rutile-type) ${ }^{1}$ is used in the DFT calculation, with lattice constant $a=b=4.615 \AA, c=3.043 \AA$, space group No. 136 (Figure $\mathrm{S} 1$ ). The exchange-correlation with the generalized gradient approximation (GGA) given by Perdew-Burke-Ernzerhof (PBE) is employed. The valence electron is $\mathrm{Mg} 2 \mathrm{~s}^{2} 2 \mathrm{p}^{6} 3 \mathrm{~s}^{2}$ and F $2 \mathrm{~s}^{2} 2 \mathrm{p}^{5}$. The plane-wave cutoff energy is set as $900 \mathrm{eV}$. Selfconsistent converged tolerance is set as $1.0 \times 10^{-6} \mathrm{eV} /$ atom. $k$-point mesh of the Monkhorst-Pack sampling is set as $5 \times 5 \times 8$, which is corresponding to $0.04 \AA^{-1}$ for each crystal axis. The empty bands number is set to be 150 , which is three times of the total valence electron number.

The band structure of $\mathrm{MgF}_{2}$ is shown in Figure $\mathrm{S} 2$. The calculated band gap is 7.287 $\mathrm{eV}$, while the experimental band gap is 10.8 or $12.4 \mathrm{eV}^{2,3}$ Here we use the band gap $10.8 \mathrm{eV}$ as the experimental value. So the scissor operator is set to be $3.513 \mathrm{eV}$ in the following optical properties calculation. The imaginary of dielectric constant given GGA calculation is plotted in Figure S3. It is agreement with the literature LDA calculation. ${ }^{4}$ However, both GGA and LDA calculation dielectric constant are less intensive than the experiment result. This is probably due to the inadequacy of the exchange-correlation model used in DFT calculation. As a result, the calculated refractive index is slightly small than that of the experimental observation. At the incident photon energy $1.164 \mathrm{eV}$, the calculated $n_{\mathrm{a}}$ and $n_{\mathrm{c}}$ is 1.3450 and 1.3573 respectively, while the experimental data is $n_{\mathrm{a}}=1.3727$ and $n_{\mathrm{c}}=1.3843$ according to the Sellmeier equation that derived from the measurement data. ${ }^{5}$ Nevertheless, it was found that the calculated dielectric constant were underestimated both for $c$ axis and $a$ axis direction. So the birefringence $\left(n_{\mathrm{c}}-n_{\mathrm{a}}\right), 0.0123$ given by the GGA calculation, is quite good agreement with the experimental birefringence $0.0115^{5,6}$ at $1.164 \mathrm{eV}$ incident photon energy. Applying the method described in the manuscript, the individual band contribution to the dielectric constant along $c$ axis and $a$ axis are given in Table 1. Using the Bader analysis (Table 2), we finally obtain the atomic contribution to the birefringence of $\mathrm{MgF}_{2}$ is $\mathrm{Mg}: 18 \%$ and $\mathrm{F}: 82 \%$. 


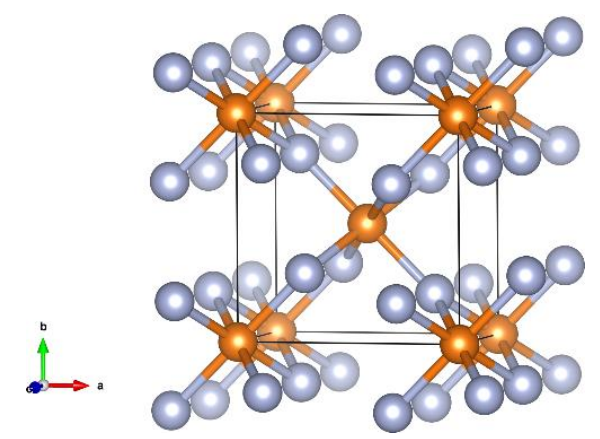

Figure S1. Crystal structure of $\mathrm{MgF}_{2}$. Color scheme: $\mathrm{Mg}$ : dark orange; F: gray.

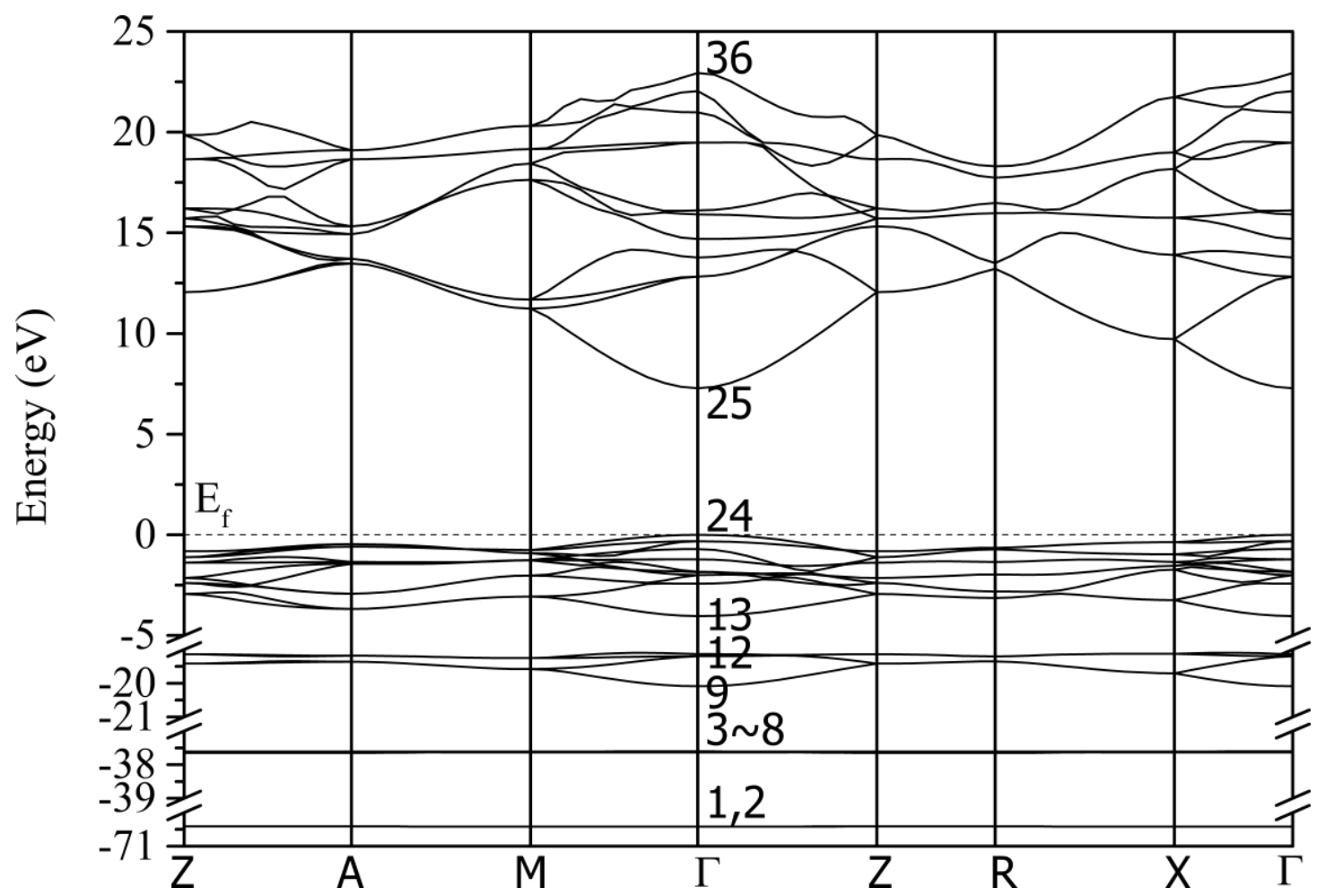

Figure S2. Band structure of $\mathrm{MgF}_{2}$. Band number is also labeled. 


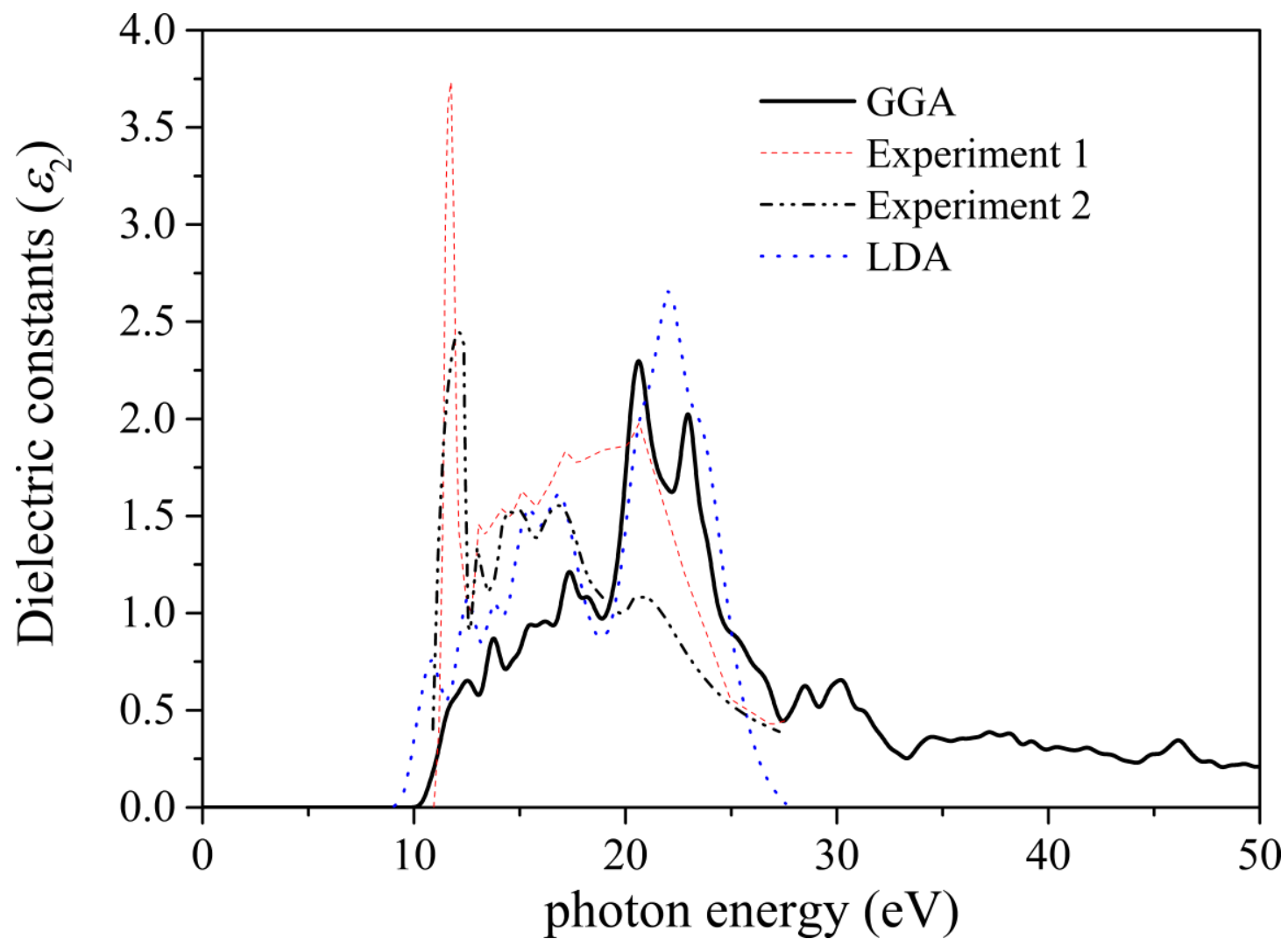

Figure S3. The imaginary part of dielectric constant $\left(\varepsilon_{a}\right)$ of $\mathrm{MgF}_{2}$. LDA is taken from ref. 4 , experiment $\mathbf{1}$ is taken from ref. 2, experimental $\varepsilon_{\mathrm{a}} \mathbf{2}$ is taken from ref. 3 .

Table 1. Band contribution (\%) to the imaginary of dielectric constant at $1.164 \mathrm{eV}$.

\begin{tabular}{lll}
\hline Band No. & a-axis direction & c-axis dire \\
Valence band & & \\
10 & 0.003 & 0.011 \\
11 & 0.015 & 0.016 \\
12 & 0.019 & 0.031 \\
13 & 1.471 & 1.576 \\
14 & 1.855 & 1.966 \\
15 & 1.857 & 2.089 \\
16 & 1.776 & 3.571 \\
17 & 3.059 & 4.710 \\
18 & 3.919 & 4.493 \\
19 & 4.918 & 4.900 \\
20 & 5.059 & 5.300 \\
21 & 4.540 & 5.807 \\
22 & 6.223 & 6.852 \\
23 & 7.817 & 3.339 \\
24 & 5.859 & 3.779 \\
Conduction band & & \\
25 & 9.268 & 8.553 \\
26 & 4.638 & 6.012
\end{tabular}




\begin{tabular}{lll}
27 & 3.190 & 4.683 \\
28 & 2.902 & 4.860 \\
29 & 2.292 & 2.035 \\
30 & 2.568 & 1.930 \\
31 & 2.927 & 1.706 \\
32 & 2.898 & 1.890 \\
33 & 2.004 & 1.612 \\
34 & 1.987 & 1.841 \\
35 & 2.023 & 1.883 \\
36 & 1.750 & 1.736 \\
37 & 1.421 & 1.330 \\
38 & 0.728 & 0.818 \\
39 & 0.680 & 1.014 \\
40 & 0.750 & 0.630 \\
\hline
\end{tabular}

Table 2. atomic Bader effective charge (e) of some of the valence and conduction bands at $\Gamma k$-point

\begin{tabular}{lll}
\hline Band No. & $\mathrm{F}$ & $\mathrm{Mg}$ \\
22 & 0.4979 & 0.0041 \\
23 & 0.4979 & 0.0041 \\
24 & 0.4997 & 0.0006 \\
25 & 0.4223 & 0.1553 \\
26 & 0.4214 & 0.1573 \\
27 & 0.4214 & 0.1573 \\
\hline
\end{tabular}

\section{References:}

1. Vidal-Valat G.; Vidal J.P.; Zeyen C.M.E.; Kurki-Suonio K., Neutron Diffraction Study of Magnesium Fluoride Single Crystals, Acta Cryst. B, 1979, 35, 1584-1590.

2. Williams M. W., Macrae R. A., Arakawa E. T., Optical Properties of Magnesium Fluoride in the Vacuum Ultraviolet, J. Appl. Phys., 1967, 38(4), 1701-1705.

3. Thomas J.; Stephan G.; Lemonnier J.C.; Nisar M.; Robin S., Optical Anisotropy of $\mathrm{MgF}_{2}$, in Its UV Absorption Region, Phys. Stat. Sol. (b), 1973, 56, 163-170.

4. Babu K. R.; Lingam C. B.; Auluck S.; Tewari S. P.; Vaitheeswaran G., Structural, Thermodynamic and Optical Properties of $\mathrm{MgF}_{2}$ Studied from First-Principles Theory, J. Solid State Chem., 2011, 184, 343350.

5. Li H. H., Refractive Index of Alkaline Earth Halides and Its Wavelength and Temperature Derivatives, J. Phys. Chem. Ref. Data, 1980, 9, 161-289.

6. Marilyn J.D., Refractive Properties of Magnesium Fluoride, Appl. Optics, 1984, 23(12), 1980-1985. 